\title{
CONTRADICCIONES NORMATIVAS: JAQUE A LA CONCEPCIÓN DEDUCTIVISTA DE LOS SISTEMAS JURÍDICOS
}

\author{
«...para trazar un límite al pensamiento tendríamos que ser capa- \\ ces de pensar ambos lados de este límite, y tendríamos por consi- \\ guiente que ser capaces de pensar lo que no se puede pensar.» \\ Ludwig Wingenstein \\ Prólogo al Tractatus Lógico-Philosophicus
}

La aplicación de los desarrollos teóricos de la lógica formal al análisis del derecho ha generado siempre fuertes reacciones, tanto a su favor como en su contra. Transitando desde el más absoluto desprecio hasta la adoración casi fetichista, y pasando por el mortal terror frente a tanto símbolo extraño, muchos no han sabido guardar la mesura y serenidad requerida para una discusión seria.

Poner un poco de orden en tan acalorado debate ha sido el propósito de quienes han buscado elucidar los límites de aplicabilidad de la lógica al terreno jurídico, cometido que constituye también el objetivo de este trabajo.

Si bien muchos críticos radicales han sostenido que el lenguaje del derecho no es susceptible de tratamiento formal, o que la lógica deductiva resulta estéril como perspectiva para el estudio de un material tan problemático como el jurídico, parece intuitivamente fuera de discusión que cuando analizamos un conjunto de normas, suponemos que el mismo comprende todas sus consecuencias lógicas.

Nadie negaría que si una norma constitucional, por ejemplo, exige una mayoría de 2/3 de los miembros del Congreso para la válida aprobación de un cierto tipo de ley, y una ley de tales características ha sido votada por $3 / 4$ de los miembros del cuerpo legislativo, dicha ley es válida.

Por cierto que afirmar la validez de la norma así votada supone extraer una consecuencia lógica del enunciado constitucional -si leyes de esta clase serán consideradas válidamente sancionadas por el Congreso cuando cuenten con el voto de 2/3 del cuerpo, entonces las que cuenten con el voto de 3/4 de sus miembros serán también válidas-, e integrar así el conjunto de los enunciados normativos explícitos de la Constitución con todos los enunciados implícitos que se deriven lógicamente de ellos. Aceptar esto, sin embargo, parece 
compelernos a conceder que los sistemas jurídicos son normalmente interpretados como sistemas deductivos, una de las más fecundas propuestas de los partidarios del análisis lógico del derecho.

2. Nuestra intención es explorar aquí una de las corrientes argumentativas de crítica a dicha concepción. Lo que resulta destacable de la misma es que, por una parte, tiene cierto aire paradójico, por cuanto supone una impugnación fundada en argumentos extraídos de la propia lógica, y por la otra, que las consecuencias negativas respecto de la concepción deductivista no parecen haber sido refutadas directamente por ninguno de los principales partidarios de esta línea de trabajo.

El argumento en cuestión consiste en una reducción al absurdo expuesta en términos similares por diversos autores ${ }^{1}$, y podría ser resumido de la siguiente manera: en el marco de la lógica deductiva, de un conjunto lógicamente inconsistente de fórmulas, cualquier fórmula es deducible. Siendo ello así, y si concebimos a los sistemas jurídicos como sistemas deductivos, habrá que aceptar que cualquier norma arbitrariamente elegida será consecuencia de un sistema jurídico inconsistente, razón por la cual el mismo no podrá cumplir con su función, esto es, dirigir la conducta de sus destinatarios, dado que en él toda conducta será obligatoria y al mismo tiempo prohibida. Ahora bien, en la mayoría de los sistemas jurídicos existentes pueden detectarse contradicciones ${ }^{2}$, pese a lo cual ningún jurista aceptaría el terrible alcance que parece predicar la lógica de tales defectos.

A partir de dichas consideraciones, cabe derivar distintas conclusiones desfavorables respecto de la concepción deductivista de los sistemas jurídicos. En lo que sigue, y para simplificar su análisis, las resumiremos en dos posiciones a las que calificaremos de tesis fuerte y tesis débil:

-(Tesis fuerte) Los sistemas jurídicos no pueden ser concebidos como sistemas deductivos. La lógica formal o deductiva aplicada al análisis del derecho lleva a consecuencias tan manifiestamente inadmisibles, que debe ser reemplazada por alguna clase de «nueva lógica».

${ }^{1}$ Véase por ejemplo, Atienza, Manuel, «Sobre los límites de la lógica en el Derecho», publicado en Theoria - Segunda época, Vol. VII, 1992, n. ${ }^{\text {ss }}$ 16-17-18, tomo B, págs. 1007-1018. Sin proyecciones críticas respecto de la concepción deductivista, el mismo problema es identificado en Lindahl, Lars, "Conflicts in systems of legals norms: A logical point of view", ponencia presentada al III Congreso de Filosofía Jurídica y Social, Asociación Argentina del Derecho, Buenos Aires, 1991.

${ }^{2}$ Las expresiones «contradicción», «inconsistencia» e «incompatibilidad lógica»serán tomadas como sinónimas a los fines del presente trabajo. 
- (Tesis débil) La concepción deductivista de los sistemas jurídicos no puede dar cuenta de ciertas intuiciones elementales de los juristas. En razón de ello, si bien la lógica formal constituye un aparato teórico sumamente poderoso para esclarecer diversos problemas de la teoría del derecho, debe ser necesariamente complementada con otras perspectivas de análisis.

Si bien podrían formularse posiciones de grado intermedio entre ambas tesis, es manifiesta la diferencia entre lo afirmado por una y otra. No sólo no es lo mismo, sino que además resultaría contradictorio afirmar, por una parte, que los sistemas jurídicos no pueden ser concebidos como sistemas deductivos, y postular asimismo que la lógica es una herramienta necesaria pero insuficiente para el análisis del derecho. Sin embargo, no falta quien, como Atienza ${ }^{3}$, afirma conjuntamente ambas cosas.

La delimitación del campo temático de la lógica ha estado siempre emparentada con la caracterización de la noción de consecuencia deductiva ${ }^{4}$. Si por «lógica» interpretamos «lógica deductiva», entonces afirmar que los sistemas jurídicos no pueden ser concebidos como sistemas deductivos vale tanto como afirmar que la lógica no tiene cabida en el derecho.

Siendo ello así, no resulta claro cómo podría el análisis lógico revestir el carácter de instrumento teórico necesario para el estudio de los sistemas jurídicos, si los mismos no pueden siquiera ser concebidos como sistemas deductivos. Porque, o bien el profesor español alude con la expresión «análisis lógico» a la lógica deductiva, en cuyo caso el señalar la fertilidad de sus aplicaciones al derecho se vuelve abiertamente contradictorio con su rechazo de la tesis deductivista, o bien el «análisis lógico» al que se refiere como «herramienta necesaria» para el estudio del derecho es un análisis lógico no deductivo, pero en tal caso reconocer la importante contribución de los desarrollos de lógica deductiva en el campo jurídico no parece más que una galantería académica sustentada en una utilización ambigua del lenguaje.

3. Estas conclusiones desfavorables en tomo a la utilización de la lógica deductiva en el campo jurídico se respaldan en la intuición corriente entre los juristas acerca de que los sistemas jurídicos, pese a la existencia de contradicciones, pueden cumplir con su función, hecho acerca del cual la lógica no podría dar cuenta.

\footnotetext{
${ }^{3}$ En el trabajo antes citado, págs. 1017 y 1018.
}

${ }^{4}$ Sobre la delimitación del campo de estudio de la lógica y la noción de consecuencia deductiva puede consultarse Alchourrón, Carlos, «Las concepciones de la lógica», inédito. 
Resaltemos inicialmente que los argumentos centrales de esta crítica parten de ciertos postulados que se encuentran fuera de toda discusión:

a) Es por cierto exacto que a partir de un sistema inconsistente, todo enunciado es derivable. Tan es así que Tarki, a fin de independizar su caracterización de la noción de consistencia respecto de la negación, la define del siguiente modo:

"A set of sentences is called consistent if it is not equivalent to the set of all meaningful sentences (or, in other words, if the set of its consequences does not contain as elements all meaningful sentences). $\|^{5}$

b) Asimismo, y en un análisis elemental, parecería correcto que la concepción deductivista lleva a aceptar que en aquellos sistemas normativos inconsistentes, toda consecuencia es derivable, y por tal motivo resultarían inservibles para cumplir con su función. Esta circunstancia es incluso identificada explícitamente por defensores del análisis lógico del derecho tan conspicuos como los profesores Carlos Alchourrón y Eugenio Bulygin:

«Si tanto una proposición p, como su negación -p forman parte del sistema, se dice que este último es incoherente. El problema con los sistemas incoherentes es que es imposible por razones lógicas satisfacer (obedecer) todas sus normas. Por lo menos las normas !p y !-p no pueden ser obedecidas ambas. Más aún, si se acepta la noción clásica de consecuencia, los efectos de una contradicción normativa son aún más desastrosos: todas las proposiciones pertenecen al sistema incoherente. Esto es así porque de acuerdo a la noción clásica de consecuencia, de un par de proposiciones contradictorias puede ser derivada cualquier proposición. De tal manera resulta que todos los sistemas incoherentes son equivalentes: ellos contienen las mismas consecuencias y son igualmente inservibles. Todo es obligatorio conforme a un sistema tal y nadie puede obedecer todas sus normas y, por lo tanto, un sistema así no puede guiar ninguna conducta» ${ }^{6}$.

${ }^{5}$ Tarski, Alfred, «Fundamental concepts of the metodology of the deductive sciences»; en Logic, Semantics, Metamathematics, Indiana, Hackett Publishing Company, 1983, pág. 90.

${ }^{6}$ Alchourrón, Carlos y Bulygin, Eugenio, «La concepción expresiva de las normas», en Análisis lógico y derecho, Madrid, Centro de Estudios Constitucionales, 1991, pág. 141, título original: «The Expresive Conception of Norms», publicado en Flilpinen, R. (Ed.), New Esays in Deontic Logic, Reidel, Dordrecht-Boston-London, 1981. 
Incluso llegan a expresar en el mismo trabajo que, debido a lo anterior, una posible resolución al conflicto entre normas puede curiosamente lograrse mediante la derogación de cualquier proposición del sistema. Y ello en razón de que, si el legislador rechaza un enunciado q cualquiera, el conjunto formado por los enunciados contradictorios $\mathrm{p}$ y $-\mathrm{p}$ es uno de los conjuntos rechazados. Por cierto que en tal caso quedaría indeterminado el sistema normativo resultante de tal acto de derogación, pero ese es otro cantar.

En un escrito posterior, Alchourrón enfoca el mismo problema de la siguiente manera:

«Supongamos que a es un conjunto inconsistente vía el conjunto de hechos F y que los enunciados descriptivos de F son verdaderos. ¿Puede a llevar a cabo su función práctica de servir de guía de comportamiento? Creo que la respuesta a esta pregunta ha de ser negativa porque el sistema ha sobre determinado el comportamiento humano, puesto que son exigidas todas las acciones $y$ omisiones. $»^{7}$

c) También debe reconocerse que resulta contraintuitivo aceptar que a partir de un sistema jurídico contradictorio, cualquier solución pueda derivarse. Claro que esta conclusión parece ser una consecuencia directa de la aceptación de uno de los principios más elementales de la lógica, cuya problematicidad es reconocida por las siguientes palabras del propio Alchourrón:

«Pocas cosas son más intuitivas que la necesidad de rechazar el principio de la lógica clásica (compartido con casi todas las lógicas salvo las lógicas relevantes y las paraconsistentes) de que de dos enunciados contradictorios (y de todo conjunto inconsistente) todo enunciado es consecuencia deductiva. Lo que se rechaza es el principio de Duns Escoto:

$$
\text { (DE) } A, \neg A \vdash B »^{8}
$$

4. De lo expuesto hasta aquí, debería al menos admitirse que la concepción deductivista de los sistemas jurídicos, y consecuente mente la plausibilidad de la aplicación del análisis lógico al derecho,

${ }^{7}$ Alchourrón, Carlos, «Conflictos de normas y revisión de los sistemas normativos», en Análisis lógico y derecho, Madrid, Centro de Estudios Constitucionales, 1991, pág. 301, título original: «Conflicts of Norms and Revision of Normative Systems», presentado en el Congreso de Miami, 1988.

${ }^{8}$ Alchourrón, Carlos, «Las concepciones de la lógica», ya citado, pág. 51. 
se ven jaqueados frente a una objeción asentada sobre cimientos cuya solidez es incluso confirmada por los destinatarios de la amenaza.

Un jugador de ajedrez amenazado de jaque en una partida no cuenta más que con tres posibles alternativas de respuesta: huir de la amenaza, bloquearla, o bien eliminar a la pieza amenazante. Cada una de tales estrategias tiene sus ventajas y sus desventajas: si el rey huye del jaque, el destino de la huida puede resultar ser más peligroso que la ubicación de origen; cuando bloqueamos el ataque, la seguridad de nuestra posición depende de la suerte de la pieza que bloquea, la cual atrae sobre sí una amenaza que perdura; finalmente, eliminar la agresión tomando la pieza que nos ataca, lo que generalmente se presenta como la respuesta más favorable, no es por cierto siempre posible.

5. En la perplejidad en la que probablemente se vería sumido un partidario de la lógica aplicada al derecho ante las críticas formuladas, podría reaccionar intentando eliminar el problema por definición: predicar la coherencia como propiedad necesaria de los sistemas jurídicos. Esta respuesta se vería respaldada por el grueso de los doctrinarios ortodoxos de la teoría del derecho, siendo Kelsen en buena parte de su obra uno de sus principales corifeos. Pero, ¿hasta qué punto esta estrategia escapista resulta conveniente? Sustentar un criterio tal nos llevaría a enervar las consecuencias teóricas más interesantes que resultan de aplicar el análisis lógico al estudio del derecho: la identificación de defectos en los sistemas jurídicos y en particular, la discriminación entre sistemas consistentes e inconsistentes.

Además, puede aquí trazarse un paralelo con una situación bastante similar y de larga polémica en la teoría jurídica: hay tanta semejanza entre los sistemas jurídicos consistentes y los inconsistentes como entre los valorados como justos y los valorados como injustos desde la perspectiva de un sistema axiológico cualquiera, por lo que vale en este punto el argumento metodológico de $\mathrm{Hart}^{9}$ basado en prescindir de dichas diferencias y abordar ambas categorías como temática común de estudio.

En definitiva, si rechazar una crítica que pretende poner en duda la aplicación de la lógica al derecho nos obliga a poner en fuga a nuestro rey, restringiendo «ad hoc» lo que entendemos por sistema jurídico, o eliminando algunas de sus más ricas conclusiones teóricas, esta ruta nos está colocando en una posición tanto o más peligrosa que aquella de la que huimos, por lo que no parece una buena jugada.

\footnotetext{
${ }^{9}$ Hart, Herbert L. A., El concepto de derecho, Buenos Aires, Abeledo Perrot, 1968, págs.
} 258 y ss. 
6. Una segunda alternativa frente a estos argumentos, bastante más plausible que la anterior, consistiría en reconocer como trivial su corrección, pero reputándolos inocuos como impugnación a la caracterización de los sistemas jurídicos como sistemas deductivos, es decir, no aceptar la extensión de las conclusiones que a partir de ello se pretende fundar.

Concedamos hipotéticamente que de hecho existen sistemas jurídicos inconsistentes. Concedemos también que, concebidos como sistemas deductivos, de los sistemas jurídicos comprensivos de enunciados contradictorios puede derivarse cualquier solución y que por lo tanto ellos no pueden cumplir su función. Concedamos incluso que esta última afirmación repugnaría la intuición de la mayoría de los juristas. ¿Lleva todo esto necesariamente a poner en tela de juicio al análisis lógico del derecho?

Podría responderse que no, señalando que la identificación de las condiciones en las que un sistema normativo es inconsistente es una tarea de la lógica. Y si aceptamos para ello a la lógica, no podemos rechazar luego las consecuencias que de tales defectos la lógica predica. Y mucho menos podemos rechazar a la misma lógica, so pretexto de las inconvenientes consecuencias lógicas que trae aparejada la noción de inconsistencia, para cuya caracterización utilizamos criterios lógicos.

Viendo así las cosas, lo que aparentaba ser una crítica sólida y convincente, parece ahora poco menos que absurda. Pero especifiquemos esta línea de análisis respecto de las dos diferentes conclusiones críticas expuestas. La tesis fuerte se proponía descartar por inservible a la lógica deductiva como perspectiva de análisis del derecho. ¿Esa es la única alternativa posible frente al problema? ¿No sería tanto o más convincente descartar por inservibles, con idéntico criterio, a los sistemas jurídicos inconsistentes, preservando la aplicabilidad de la lógica?

La lógica nos permite detectar algunos de los defectos que aquejan a los sistemas jurídicos, entre ellos la inconsistencia. De tal manera, suministra un criterio negativo de adecuación para los sistemas normativos, pues un sistema inconsistente requiere modificaciones sustanciales si ha de ser útil como guía de la conducta ${ }^{10}$. En otras palabras, la lógica permite identificar incoherencias en los sistemas normativos y a partir de sus postulados es posible advertir la inutilidad práctica de tales sistemas. Descalificar al análisis lógico porque pone en evidencia una consecuencia desagradable como ésta, sería tanto como matar al mensajero que nos comunica malas noticias.

${ }^{10}$ Véase Alchourrón, Carlos, «Conflictos de normas y revisión de sistemas normativos», ya citado, págs. 291 y 292. 
O más aún: sería como descalificar a la ciencia médica porque un oncólogo nos diagnostica un cáncer. Lo que corresponde es reconocer que los sistemas jurídicos inconsistentes deben ser modificados si se pretende que cumplan con su función de guía de la conducta.

De acuerdo con la tesis débil, la lógica no puede dar cuenta de fuertes intuiciones de los juristas. Frente a ello, se propone sacrificar a la lógica, considerándola herramienta insuficiente para el análisis del derecho. Como en el caso anterior, cabe aquí otro camino: centrar el problema no en la lógica, sino en la imposibilidad de reconstruir coherentemente las intuiciones de los juristas.

En un trabajo ya citado de Alchourrón ${ }^{11}$, luego de reconocerse la contraintuitividad del principio de Duns Escoto, se expresa:

"Igualmente es muy difícil no admitir la enorme intuitividad de la regla de Introducción de la Disyunción (I. $\vee .1)[A>(A \vee B)]$, el principio de monotonía $(\vdash$.3) (si $\alpha \vdash$ A entonces $\alpha U \beta \vdash A)$, el principio del Silogismo Disyuntivo:

$$
\text { (SD) } \neg A,(A \vee B) \vdash B
$$

$y$ de la suerte de transitividad de la noción de consecuencia representada por la regla de Corte ( $\vdash$.2) (si $(\alpha \vdash B y \alpha U\{B\} \vdash A$ entonces $\alpha \vdash A)$.

Lamentablemente todas estas fortísimas intuiciones no pueden convivir coherentemente ya que la aceptación de los cuatro últimos principios comprometen a la aceptación del principio de Duns Escoto. La siguiente es una derivación de (DE) a partir de los otros cuatro principios indicados:
(1) $A, \neg A \vdash(A \vee B)$
[De (I. v.]) por (เ. 3)]
(2) $\mathrm{A}, \neg \mathrm{A},(\mathrm{A} \vee B) \vdash B$
$[D e(S D)$ por (เ.3)]
(3) $A, \neg A \vdash B$
[De (1) y (2) por (เ. 2)]

La más leve aspiración de coherencia nos compele a rechazar alguna de estas sólidas intuiciones, pero por otro lado no hay base intuitiva para el sacrificio de ninguna.»

La conclusión de esta sencilla y paradójica demostración de Alchourrón -similar a la utilizada por Lewis para defender su con-

${ }^{11}$ Alchourrón, Carlos, «Las concepciones de la lógica», ya citado, págs. 51 y 52. 
cepción de la implicación estricta ${ }^{12}$ - es que no se puede dar satisfacción a todas nuestras intuiciones corrientes. A efectos de proyectar esta idea con relación a las intuiciones de los juristas, sería necesario contar previamente con una caracterización adecuada de la noción de contradicción normativa. Bástenos, por ahora, con señalar que no constituye en absoluto una objeción válida contra la aplicación de la lógica al derecho el que la misma no brinde una reconstrucción completa y coherente de las intuiciones de los juristas. Esto en la medida que no se nos ofrezca otro sistema en su reemplazo que sí garantice tal cometido, pues existe -al menos- la posibilidad de que ninguna reconstrucción coherente de todas las intuiciones jurídicas pueda alcanzarse.

7. En el mismo plano que la tentativa anterior habría que ubicar la respuesta consistente en aceptar las objeciones planteadas, pero rescatando sin embargo la deducibilidad aplicada al derecho como un ideal racional.

Moreso y Navarro ${ }^{13}$ consideran que el criterio de deducibilidad es demasiado amplio como para identificar la pertenencia de normas a un sistema, ya que entonces cualquier sistema jurídico contendría infinitas normas y, en casos de inconsistencia, todas las normas pertenecerían al mismo.

No obstante lo anterior, se sostiene, cabría interpretar que el criterio de deducibilidad, conjuntamente con otros, si bien no sirve para decidir la pertenencia de normas a un sistema jurídico real, si permite definir un «orden jurídico racional». Dicho criterio, al igual que la completitud, la coherencia, etc., cumpliría entonces el rol de un «ideal racional» ${ }^{14}$.

Esta postura se presenta como más endeble que la anterior ya que coloca en un mismo nivel a la noción de deducibilidad y a las de completitud y coherencia. Ello parece incorrecto toda vez que las dos últimas se definen en términos de la primera: es imprescindible una caracterización previa de la noción de consecuencia deductiva a fin de poder introducir los otros dos conceptos. Por lo demás, si recurrimos al sutil artilugio de apelar a un «legislador racional», es debido a lo contraintuitivas que resultan ciertas aplicaciones de la

${ }^{12}$ Lewis, C. L., A Survey of Simbolic Logic, California University Press, 1918, citado por Haack, Susan, Filosofía de las lógicas, Madrid, Cátedra, 1982, pág. 223.

${ }^{13}$ Moreso, José Juan y Navarro, Pablo E., «Algunas observaciones sobre las nociones de orden jurídico y sistema jurídico», en Análisis Filosófico, volumen XII, numero 2, noviembre de 1992, pág. 142. 1983.

${ }^{14}$ Von Wright, G., «Norms, Truth and Logic», en Practical Reason, London, Basil Blackwell, 
lógica en el terreno de las normas. Sin embargo, la misma idea de un legislador racional es bien poco intuitiva ${ }^{15}$.

De cualquier modo, la principal objeción que podría dirigirse contra estas últimas tentativas de respuesta no se refiere al contenido de lo afirmado, sino más bien a la estrategia utilizada. Ocurre que cuando aceptamos las premisas del razonamiento crítico pero rechazamos su conclusión, arguyendo alternativamente que el problema no está en la lógica, sino en los sistemas inconsistentes, en las intuiciones de los juristas, o que el análisis lógico puede preservarse como una «lógica para un legislador racional», no damos respuesta a la cuestión de fondo. Habremos bloqueado momentáneamente la amenaza descalificadora de la aplicación de la lógica al derecho, pero seguirá pendiendo sobre nuestras cabezas la espada de la crítica.

La mayoría de los juristas estarían dispuestos a sostener que la existencia de contradicciones en un sistema jurídico no impide que éste pueda cumplimentar su cometido, al menos en alguno de los sentidos que podrían asignarse a dicha expresión. En la medida en que el análisis lógico no pueda dar cuenta de esta intuición, por más que resaltemos que al menos la lógica nos permite identificar los sistemas inconsistentes y las consecuencias de tal defecto, o que algunas de las intuiciones de los juristas son incompatibles entre sí, o que las conclusiones de la aplicación del análisis lógico valen para la hipótesis de un utópico legislador racional, es claro que el valor del modelo propuesto para dar cuenta de los problemas de la teoría del derecho se ve relativizado. Así, los movimientos que bloquean el jaque parecen también inadecuados porque conceden demasiada ventaja al rival.

8. Quedaría por explorar la última opción: ¿es posible tomar la pieza que amenaza de jaque al rey deductivista? ¿Puede darse una respuesta satisfactoria desde la lógica al problema de fondo presupuesto por las críticas esbozadas?

Para eso sería menester concentrarse en las premisas del argumento que estamos analizando, y rechazar lisa y llanamente o reinterpretar el alcance de una o varias de ellas.

Está fuera de toda cuestión que cualquier enunciado es consecuencia de un sistema deductivo inconsistente. Tampoco ofrece reparos el hecho de que existan sistemas jurídicos que contengan enunciados contradictorios. Sin embargo, lo que afirman las restantes premisas debe ser estudiado con detenimiento, por cuanto podríamos vernos inducidos a error a partir de formulaciones imprecisas.

${ }^{15}$ Con respecto a este argumento, véase Alchourrón, Carlos y Martino, Antonio, «Lógica sin verdad», en Theoria - Segunda época, año III, 1987-88, n. ${ }^{\text {os }-7-8, ~ p a ́ g . ~} 23$. 
En lo que sigue, intentaremos demostrar: a) que resulta ambiguo afirmar que de los sistemas jurídicos comprensivos de enunciados contradictorios puede deducirse cualquier norma, tanto como sostener que los sistemas jurídicos con enunciados contradictorios resultan inservibles para el cumplimiento de su función, y b) que las elementales intuiciones de los juristas no sólo no se oponen, sino que más bien reafirman las conclusiones teóricas de la aplicación del análisis lógico al derecho, a poco que se supere un estadio meramente superficial.

9. A los fines propuestos en el punto anterior, un primer esclarecimiento conceptual se impone: ¿qué debemos entender por contradicción normativa?

9.1. La respuesta no es en absoluto sencilla, dado que a su vez presupone al menos uno de los problemas que más tinta han hecho correr entre quienes se empeñan en aplicar el análisis lógico al derecho: el de la posibilidad de asignar valores veritativos a las normas.

La función que cumple el lenguaje cuando emitimos una prescripción es distinta de aquella que cumple cuando describimos un cierto estado de cosas, y sólo cuando el lenguaje es utilizado en función descriptiva es que podemos calificar de verdadero o falso lo que decimos. Si las normas no son susceptibles de ser calificadas como verdaderas o falsas, entonces o bien la noción de inferencia y las conectivas lógicas se caracterizan en función de las nociones de verdad o falsedad, y entonces no habrá relaciones lógicas entre normas ni podrán aplicarse las conectivas lógicas a ellas, o bien hay una lógica de normas, pero entonces ni la noción de inferencia ni las conectivas lógicas deben ser caracterizadas apelando a las nociones de verdad o falsedad. Tal, la dilemática enunciación de Jorgersen ${ }^{16}$.

Los caminos tradicionales de investigación en tomo a la lógica del discurso normativo han apuntado a tomar el primer cuerno del dilema: asignar de algún modo valores de verdad, o algún sucedáneo, a las normas. La alternativa, mucho menos desarrollada, ha consistido en buscar alguna noción más general, como una caracterización abstracta de consecuencia deductiva, a fin de adoptarla como término primitivo de la lógica, posibilitando así una lógica de normas sin verdad ${ }^{17}$.

16 Jorgensen, J., «Imperatives and logic», en Erkenntnis, tomo 7, 1937/38, citado por Alchourrón, Carlos y Martino, Antonio, «Lógica sin verdad», ya citado.

${ }^{17}$ Sobre las distintas perspectivas seguidas por los teóricos puede consultarse el trabajo citado en la nota anterior, así como la Introducción de Análisis lógico y Derecho, también citado. 
9.2. Sin inmiscuirnos en este problema por exceder el marco de nuestro trabajo, es evidente que, si algo hay a lo que podamos calificar como «contradicción entre normas», el sentido de dicha expresión no puede ser idéntico al de la contradicción entre proposiciones.

El paradigma de lo que los lógicos interpretan por contradicción normativa está representado por el par deóntico Op y $\mathrm{O}$ - p, esto es, que sea ordenada una conducta y su abstención -o, lo que es lo mismo, que una conducta sea ordenada y prohibida-. Si bien nadie dudaría que estas normas son contradictorias, sí en cambio sería problemático intentar explicar por qué ambas resultan incompatibles.

Por lo pronto, no podría decirse que son contradictorias porque no pueden ser ambas verdaderas, pues no cabe hablar de la verdad o falsedad de tales enunciados. No sirve tampoco el criterio de hacer derivar la incompatibilidad del hecho de que no puedan coexistir ambas en un conjunto de normas ${ }^{18}$. En realidad nada impide que un legislador ordene un acto y su abstención, de manera que sí pueden coexistir ${ }^{19}$. Lo que se quiere decir cuando se habla de la imposibilidad de coexistencia en el marco de un conjunto de normas, es que ambas no pueden coexistir coherentemente, con lo cual se presupone lo que se quiere definir, cayéndose en una petición de principio.

9.3. Una respuesta que se ha tentado para explicar el problema consiste en situarse en la perspectiva del sujeto destinatario de las normas, identificando la contradicción normativa con la imposibilidad lógica de cumplimiento.

Afirmar que dos normas son contradictorias cuando un sujeto no puede satisfacerlas ambas al mismo tiempo por razones lógicas, trae aparejado el problema de que en tal caso, lo que se está afirmando es que un sujeto no puede al mismo tiempo hacer verdadera la proposición p y la proposición -p, esto es, los contenidos de las normas contradictorias. Con ello, se está brindando un criterio para identificar contradicciones entre contenidos normativos -no pueden ser verdaderas $\mathrm{p}$ y $-\mathrm{p}$-, pero no se explica en definitiva por qué las normas son incompatibles. Que la contradicción entre contenidos normativos es condición necesaria pero insuficiente para hablar de contradicción entre normas lo pone de manifiesto el que dos normas permisivas de un acto y su abstención, Pp y P-p, no implican contradicción alguna.

Esa última objeción, fundada en el carácter deóntico de las normas, resalta la necesidad de asumir tal componente como un ingre-

${ }^{18}$ Von Wright, Georg H., Norma y Acción, Madrid, Tecnos, 1970, pág. 148.

${ }_{19}$ Acerca de las críticas a ambas ideas, véase Alchourrón, Carlos y Bulygin, Eugenio, «Fundamentos pragmáticos para una lógica de normas», en Análisis lógico y derecho, ya citado, pág. 160. 
diente cuya relevancia no puede ser obviada al momento de caracterizar la noción de contradicción normativa, si reputamos al acto de permitir como un acto lingüístico diverso del de obligar. En tal caso, no sólo habrá contradicción cuando una autoridad haya ordenado y prohibido un mismo acto, sino también cuando lo haya permitido y prohibido.

A efectos de lograr una adecuada reconstrucción de la noción de contradicción entre normas, deberían entonces establecerse criterios específicos para identificar contradicciones respecto de conjuntos de normas de obligación, conjuntos de normas permisivas y conjuntos mixtos de normas imperativas y permisivas. Alchourrón ${ }^{20}$ indica que un conjunto de normas imperativas sería consistente si, y sólo si, hubiera una interpretación tal que hiciera verdaderos todos los enunciados de acción que siguen al operador deóntico «O»; un conjunto de normas permisivas sería consistente si, y sólo si, para cada uno de los enunciados de acción que siguen inmediatamente al operador deóntico «P» hubiese una interpretación que hiciera verdaderos a cada uno de ellos; y finalmente, un conjunto mixto de normas imperativas y permisivas sería consistente si, y sólo si, para cada enunciado permisivo hubiera una interpretación que hiciera verdadero su enunciado de acción y también hiciera verdaderos a todos los enunciados de acción contenidos en los enunciados imperativos del conjunto.

9.4. Otra alternativa teórica ideada para superar la mera reducción de las contradicciones normativas a la incompatibilidad entre sus contenidos proposicionales, ha reivindicado la necesidad de situar el análisis desde el plano de la autoridad normativa y, con criterio pragmático, señalar que existe contradicción toda vez que hay discordancia entre la intención de ordenar y su expresión verbal ${ }^{21}$.

Lo normal al ordenar una cierta conducta es que la intención de la autoridad normativa sea que el acto en cuestión se realice. Así, habría contradicción entre dos normas para una autoridad normal cuando sus expresiones lingüísticas (por ejemplo, Op y O - p) frustren tal intención genérica.

Esta respuesta tiene dos problemas: el primero es que, así como nada obsta a que de hecho un legislador ordene un acto y su abstención -razón por la que no puede decirse que dos normas sean incoherentes si no pueden coexistir en un conjunto-, nada impide tampoco que un legislador quiera que se realice una acción y también quie-

${ }^{20}$ Alchourrón, Carlos, «Conflictos de normas y revisión de los sistemas normativos», ya citado, pág. 295.

${ }^{21}$ Alchourrrón, Carlos y Bulygin, Eugenio, «Fundamentos pragmáticos para una lógica de normas», ya citado, pág. 160 y 161 . 
ra que no se realice: cualquier autoridad real puede tener intereses o voliciones conflictivas. Hasta podría pensarse en el caso de que el legislador tuviese el malicioso, pero perfectamente coherente interés de normar exprofeso incoherentemente una conducta, a fin de poder luego resolver en uno u otro sentido de acuerdo con lo que le resulte circunstancialmente más conveniente.

Esto obligaría a buscar un posible escape a tales objeciones haciendo referencia a la intención de un «legislador normal». Pero la noción de un «legislador normal» conlleva los mismos reparos que pueden dirigirse contra la del «legislador racional $»^{22}$.

Si bien por las razones someramente expresadas no parece satisfactoria la postura anterior como criterio para la identificación de las contradicciones entre normas, es útil para remarcar la relativa dependencia de la noción de contradicción normativa con relación a la autoridad que emite las normas. Sumando esto a la posibilidad de cumplimiento por parte del destinatario resulta que dos normas emitidas por la misma autoridad pueden ser contradictorias respecto de un sujeto y no serlo respecto de otro, como así también, que dos normas dictadas por diferentes autoridades normativas sean incompatibles entre sí. En el primer caso, si un sujeto $\mathrm{x}$ establece las siguientes dos normas:

$\mathrm{N} 1$ : «Es obligatorio para $y$ y para $z$ realizar el acto $\mathrm{p} »$.

N2: «Es obligatorio para $y$ abstenerse de realizar el acto p»,

es evidente que este conjunto de normas es inconsistente respecto de $y$, pero es perfectamente coherente respecto de $z$, o en otras palabras, la autoridad normativa habrá en este caso normado consistentemente la conducta de $z$ pero inconsistentemente la conducta de $y$.

En el segundo caso comentado, si un sujeto $x$ ordena a un sujeto $y$ la realización de un cierto acto $\mathrm{p}$, y un sujeto $z$ ordena a $y$ abstenerse del mismo acto $\mathrm{p}$, en tal caso, pese a que, tanto el conjunto de las normas dictadas por $x$ como el conjunto de las normas dictadas por $y$, considerados independientemente uno del otro, pueden ser cada uno perfectamente consistente (justamente porque son lógicamente independientes), el conjunto que resulta de la unión de los conjuntos de normas dictadas por $x$ y por $y$ en relación al sujeto $z$ es inconsistente.

En los sistemas jurídicos no hay uno, sino una pluralidad de individuos u órganos legitimados para el dictado de normas, aún cuando pueda considerarse metafóricamente que todos ellos actúan en función de una única autoridad normativa (el Estado). El caso de contradicción más frecuente es aquél en el cual una autoridad emite una

${ }^{22}$ Véase nota 14. 
prescripción incompatible con respecto a otra dictada por una autoridad distinta. Los juristas utilizan, para la resolución de conflictos entre normas emitidas por diferentes autoridades del sistema, el criterio de que la norma dictada por la autoridad superior prima sobre aquella que ha sido emitida por la autoridad inferior. Todo sistema jurídico establece -explícita o implícitamente- jerarquías entre sus normas, siendo la autoridad de la cual emanan uno de los factores que se toma en cuenta a tales fines. La existencia de estos criterios para la resolución de las contradicciones entre las normas de los sistemas jurídicos demuestra, no sólo que las contradicciones entre normas existen, sino además que esto es vivido por los juristas como algo intolerable.

9.5. A lo dicho hasta aquí resulta casi superfluo agregar que también deben considerarse las circunstancias de tiempo y lugar en las cuales las normas exigen o permiten realizar o abstenerse de realizar ciertos actos, a efectos de una acabada delimitación de la noción de contradicción normativa. Sólo habrá incompatibilidad entre dos normas cuando exista identidad en la ocasión, como se daría en el caso de que ambas exigieran la realización y la abstención de una misma conducta al mismo tiempo y en el mismo lugar.

9.6. Podríamos contentarnos con una reconstrucción de la noción de contradicción normativa en términos de la imposibilidad por parte de un sujeto de dar satisfacción a todos los contenidos normativos de las normas de obligación y a cada uno de los contenidos normativos de las normas permisivas de un sistema de normas, dictadas por una cierta autoridad y con relación a una cierta ocasión. Pero ocurre que hay una gran cantidad de casos en los cuales normas aparentemente no contradictorias provocan «conflictos normativos $\gg{ }^{23}$ significativos. Supongamos que en un sistema coexisten las siguientes dos normas:

N1: «Es obligatorio que los automovilistas se detengan frente a los semáforos en rojo.»

N2: «Está prohibido que los automovilistas se detengan junto a zonas militares.»

${ }^{23}$ La diferenciación entre las contradicciones o inconsistencias normativas y los «conflictos normativos» fue introducida por Risto Hilpinen en «Conflicts and Change in Normative Systems», en Frändberg, A. y Van Hoecke, M. The Structure of Law, Iustus Förlag, Uppsala, 1987. 
¿Hay contradicción entre ambas? Podríamos formalizar dichos enunciados de la siguiente manera:

$$
\begin{aligned}
& \mathrm{N} 1: \mathrm{A}>\mathrm{Op} \\
& \mathrm{N} 2: \mathrm{B}>\mathrm{O}-\mathrm{p}
\end{aligned}
$$

(Entendiendo por A el hecho de que los automovilistas se encuentren frente a semáforos en rojo, B como la circunstancia de que los automovilistas se encuentren junto a zonas militares y Op y $\mathrm{O}$ - p como la obligación y la prohibición de detenerse, respectivamente). Así interpretadas estas normas no parecen contradictorias.

Pero eso no quita que pueda generarse, para quien debe cumplirlas, una situación conflictiva indiscernible de la contradicción canónica entre Op y O - p. Bastaría para ello con que a alguna autoridad se le ocurriese la poco brillante pero perfectamente posible idea -llevada a la práctica en la entrada de la Base Naval de Mar del Plata- de ubicar un semáforo junto a una zona militar.

Lo que muestra el ejemplo es que dos normas pueden resultar incompatibles ante el acaecimiento de determinadas circunstancias de hecho. En nuestro caso, si se dieran conjuntamente las condiciones de hecho A y B -semáforo rojo y zona militar-, resultaría obligatorio y a la vez prohibido detenerse (Op y O - p). He aquí un nuevo elemento cuya consideración resulta imprescindible a fin de brindar una satisfactoria caracterización de las contradicciones normativas: un conjunto de normas puede ser consistente vía determinados hechos, esto es, ante el acaecimiento de determinadas circunstancias fácticas, e inconsistente vía otros hechos ${ }^{24}$.

9.7. Todas las observaciones antecedentes podrían ser abreviadas en lo siguiente: cuando afirmamos que dos enunciados (descriptivos o prescriptivos) son contradictorios, estamos formulando una aserción acerca de enunciados, esto es, una aserción metalingüística. En el caso de la contradicción proposicional, se tratará de una expresión metalingüística respecto de un lenguaje objeto descriptivo, mientras que, en el caso de la contradicción normativa, estaremos en presencia de una expresión metalingüística acerca de un lenguaje objeto prescriptivo.

Una de las diferencias existentes entre los sistemas tradicionales de lógica de normas y los sistemas de lógica de proposiciones normativas -y con ello entre las interpretaciones prescriptiva y descrip-

${ }^{24}$ Alchourrón, Carlos, «Conflictos de normas y revisión de sistemas normativos», ya citado, págs. 300 y 301 . 
tiva de los enunciados normativos-, es la presencia en los primeros del axioma:

$$
(\mathrm{LD})-(\mathrm{Op} \wedge \mathrm{O}-\mathrm{p})
$$

y su correlativa ausencia en los segundos. No obstante, dicho axioma guarda una importante analogía estructural con la definición de coherencia normativa de los sistemas de lógica de proposiciones normativas:

$$
\text { (Df.) } \operatorname{Coh}(p) \equiv-(O p \wedge O-p)
$$

De conformidad con el primero, se excluye la posibilidad de que se den conjuntamente Op y O - p, mientras que la definición de coherencia solamente determina las condiciones en que los sistemas son coherentes, pero sin excluir la posibilidad de sistemas incoherentes ${ }^{25}$.

Y hay algo todavía más importante. Cuando un sujeto le dice a otro que está lloviendo, ese enunciado («está lloviendo») se refiere a la lluvia y será verdadero o falso según que efectivamente esté lloviendo o no. Pero si un tercer sujeto, comentando la conversación sostenida por los dos anteriores, dijese "Alguien dijo que estaba lloviendo», este enunciado no se refiere a la lluvia en forma directa como el anterior, sino más bien a lo dicho por alguien. Y será verdadero o falso según que exista o no un sujeto que haya dicho realmente que estaba lloviendo ${ }^{26}$. En tal sentido, la aserción metalingüística es relativa a la existencia de un sujeto que emitió el enunciado en cuestión, lo cual no ocurre en el nivel del lenguaje objeto.

Por cierto que la afirmación «Alguien dijo que estaba lloviendo» no es en absoluto la única forma posible de describir lo ocurrido. Nuestro aprendiz de chismoso podría ser un poco más generoso con la información y decir: «José dijo que estaba lloviendo». En tal caso, su descripción será relativa a lo dicho por José, y resultará verdadera o falsa según que exista o no un sujeto que haya expresado el enunciado en cuestión y según que ese sujeto sea o no José.

${ }^{25}$ Véase Alchourrón, Carlos y Bulygin, Eugenio, «Fundamentos pragmáticos para una lógica de normas», ya citado, págs. 161 y 162 .

${ }^{26}$ Alchourrón utiliza un argumento similar, pero restringe la relatividad exclusivamente a una cierta autoridad normativa. Por las razones que se exponen, pensamos que tal criterio es injustificadamente limitado. Véase Alchourrón, Carlos, «Philosophical Foundations of Deontic Logic and the Logic of Defeasible Conditionals», en Meyer, J. J. y Wieringa, R.J., Deontic Logic in Computer Science: Normative System Specification, Wiley \& Sons, 1993, pág. 19. 
Se podría seguir agregando información (por ejemplo, «En las circunstancias tales y cuales, José le dijo a Pedro que en ese momento y en ese lugar estaba lloviendo») y, a mayor generosidad en ella, la verdad del enunciado metalingüístico resultará relativa con respecto a una mayor cantidad de factores. Lo cierto es que no existe límite alguno a la cantidad de elementos que pueden ponerse en juego.

Es importante remarcar con esto otra diferencia entre la interpretación prescriptiva y la interpretación descriptiva de los enunciados normativos. La primera asume un rasgo absoluto frente a la necesaria relatividad de la segunda. Tal relatividad dependerá al menos de la existencia de alguna autoridad normativa que haya emitido la prescripción en cuestión.

Si nuestra intención es lograr una adecuada caracterización de la noción de contradicción o inconsistencia entre normas (noción metalingüística por excelencia), habrá que hacerse cargo de su carácter relativo. A priori no existe límite alguno, como se dijo, respecto de los factores a considerar. En otras palabras, podríamos relativizar nuestra definición de contradicción entre normas tanto como quisiéramos. Hemos tomado aquí en cuenta la incidencia de factores tales como la autoridad normativa, el sujeto destinatario, la ocasión y las circunstancias de hecho, en la identificación de la incompatibilidad entre dos normas $^{27}$.

Pero no es necesario ir tan lejos para mostrar la falacia que se esconde detrás de la afirmación de que en todo sistema jurídico inconsistente resultan obligatorias y prohibidas todas las conductas. Alcanza con apreciar la naturaleza metalingüística del concepto de contradicción normativa, su carácter intrínsecamente relativo, y analizar someramente la incidencia de uno de los factores que relativizan esta noción, cuya relevancia en el derecho asume un rol definitorio.

10. Existen muchas formas posibles de representar los enunciados de un sistema jurídico. Si bien más de un teórico se ha esforzado por alcanzar «la» representación formal más adecuada de las normas jurídicas, tal representación única no existe.

Si por formalización interpretamos alguna clase de actividad de traducción a través de la cual un texto, ordenado sintácticamente de acuerdo al menos con las reglas de un cierto lenguaje, es transformado en otro conjunto de cadenas de signos con algún propósito teorético o pragmático, es evidente que, siendo diversos los objetivos del emprendimiento, una oración puede correlacionarse no con una correspondiente expresión formalizada, sino más bien con un con-

${ }^{27}$ Sobre la relatividad del concepto de inconsistencia normativa, véase Von Wright, Georg H., Norma y Acción, ya citado, págs. 159 y ss. 
junto abierto de expresiones, pudiendo todas ellas ser consideradas como sus adecuadas formalizaciones $^{28}$. Por supuesto que, asumida que sea una cierta finalidad, ella permitirá valorar ciertas formalizaciones como más adecuadas que otras.

Cuando lo buscado es una representación formal de los enunciados de un sistema normativo que posea mayor exactitud que su formulación original, pero la suficiente riqueza como para poder dar cuenta de algunos de los problemas que aquejan a los estudiosos del derecho, la extensión del explicatum, aún cuando no será idéntica a la del explicandum -pues en tal caso perdería su mayor exactitud-, deberá acercársele lo más posible ${ }^{29}$.

La mayor parte de los sistemas de lógica aplicada al discurso prescriptivo han sido ideados, tanto en sus versiones de lógicas de normas como en las de lógicas de proposiciones normativas, para dar cuenta de los enunciados prescriptivos categóricos. Con ello, una de sus principales falencias en lo que hace a la aplicabilidad práctica de sus derivaciones en relación al derecho, ha sido la imposibilidad de dar cuenta satisfactoriamente de la gran diversidad de enunciados presentes en los sistemas jurídicos, y particularmente de aquéllos formulados en términos condicionales.

Es más: la mayoría de los filósofos del derecho admitiría sin dudar la afirmación de que las normas generales contenidas en los sistemas jurídicos, tienen, como los juicios universales aristotélicos, carácter general y condicional ${ }^{30}$. Las obligaciones y derechos no son establecidos por el legislador en forma categórica, sino subordinados al cumplimiento de ciertas condiciones de hecho.

En la medida en que podamos contar con una lógica que reconstruya adecuadamente las relaciones entre enunciados normativos condicionales, podremos también satisfactoriamente dar cuenta de los enunciados normativos categóricos, entendidos entonces como prescripciones para toda circunstancia posible o, en otros términos, prescripciones con relación a un conjunto vacío de hechos ${ }^{31}$.

${ }^{28}$ Vernengo, Roberto, «Formalization in Legal Languages», en Martino, A. A., y Socci Natali, F. (Eds.), Logic, Informatics, Law, North-Holland, Elsevier Science Publishers, 1986, págs. 555 y ss.

${ }^{29}$ Acerca de las características del proceso de reconstrucción racional de conceptos, véase Alchourrón, Carlos y Bulygin, Eugenio, Introducción a la metodología de las ciencias jurídicas y sociales, Buenos Aires, Astrea, 1975, pág. 30, traducción de los autores del original Normative Systems, Viena, Springer-Verlag, 1971.

${ }^{30}$ Alchourrón, Carlos, «Philosophical Foundations of Deontic Logic and the Logic of Defeasible Conditionals», ya citado, pág. 15.

${ }^{31}$ Alchourrón, Carlos, «Conflictos de normas y revisión de sistemas normativos», ya citado, págs. 300 y 301 . 
Tal ha sido la preocupación de quienes han buscado reducir los enunciados normativos a su forma normal, lo que implica transformarlos en expresiones condicionales en las que se distingue con precisión entre las condiciones fácticas y las consecuencias normativas $^{32}$. También éste ha sido el propósito que se evidencia en los más recientes trabajos de Alchourrón ${ }^{33}$. En ellos se advierten las dificultades de la cuestión tratada: lo problemático no es reconstruir las condiciones positivas exigidas por las normas para el surgimiento de obligaciones o derechos, sino las condiciones negativas. Una acción puede ser exigida como obligatoria dada cierta situación de hecho, pero dejar de serlo en otra. Así, si volvemos al ejemplo del conductor del vehículo que se enfrenta a las dos normas contradictorias comentadas en el punto 9.6., podríamos interpretar esas prescripciones de modo tal que la obligación genérica de detenerse frente a los semáforos en rojo cediera en el caso particular de colisión con la obligación de proseguir la marcha junto a una zona militar. En tal intelección, el sistema integrado por dichos enunciados debería ser reconstruido del siguiente modo:

$$
\begin{aligned}
& \mathrm{N} 1:(\mathrm{A} \wedge-\mathrm{B})>\mathrm{Op} \\
& \mathrm{N} 2: \mathrm{B}>\mathrm{O}-\mathrm{p}
\end{aligned}
$$

N1 nos dice que si un automovilista se encuentra frente a un semáforo en rojo, debe detenerse, salvo que conjuntamente ocurra que se encuentra junto a una zona militar. N2 expresa que si el conductor se encuentra junto a una zona militar, entonces debe proseguir su marcha.

Reformular así las normas del sistema elimina la indeseada contradicción, pero ello sólo es posible mediando la información adicional de que una de las normas prima sobre la otra. Por otra parte, aún contando con dicha información, sería menester, a fin de formalizar cada enunciado del sistema, tomar en consideración todas las restantes normas y revisar la formalización de cada una toda vez que el sistema se modifique ${ }^{34}$.

El problema de la permanente revisión podría obviarse apelando

${ }^{32}$ Vernengo, Roberto, «Formalization in Legal Languages», ya citado, pág. 561.

${ }^{33}$ Pueden consultarse en particular, «Conflictos de normas y revisión de sistemas normativos», «Condicionalidad y la representación de las normas jurídicas», «Phiiosophical Foundations of Deontic Logic and the Logic of Defeasible Conditionals», ya citados y «Defeasible Conditionals as General Conditionals Plus Revision Theory», inédito.

${ }^{34}$ Véase por ejemplo, Alchourrón, Carlos, «Condicionalidad y la representación de las normas jurídicas», ya citado, pág. 269. 
a alguna otra conectiva que no fuese la implicación material, y para la cual no valgan ni la ley de refuerzo del antecedente:

$$
[(P>q)>((p \wedge r)>q)]
$$

ni la ley del modus ponens:

$$
[((P>q) \wedge p)>q]
$$

Sobre la base de la idea de las obligaciones prima facie de Ross, podría trabajarse con algún tipo de conectiva -como los llamados condicionales derrotables- que cumplieran estas dos condiciones. Ello permitiría eludir consecuencias indeseadas como las contradicciones entre normas del tipo de las del ejemplo anterior, pero al precio de perder también buena parte de las consecuencias deseadas ${ }^{35}$. Como puede verse, ninguna de las alternativas está libre de crítica.

11. Aún con todos los obstáculos que parecen complicar este derrotero, resulta mucho más adecuado representar los enunciados de un sistema jurídico en términos condicionales que categóricos.

Ya en Normative Systems ${ }^{36}$, Alchourrón y Bulygin predicaban el carácter relacional de los sistemas jurídicos, entendiendo que su función consiste básicamente en establecer correlaciones deductivas entre casos (descripciones de circunstancias de hecho) y consecuencias normativas. Se expresaba en ese trabajo que un sistema normativo $\alpha$ es coherente en relación a un universo de casos UCi si, y sólo si, ningún elemento del universo de casos UCi es correlacionado por $\alpha$ con todas las soluciones ${ }^{37}$.

$\mathrm{Si}$ bien es teóricamente concebible la hipótesis de normas categóricas, y correlativamente, una noción de contradicción normativa absoluta con relación a las circunstancias, no es ese el tipo de normas ni el tipo de contradicciones con las que tienen que habérselas los juristas.

La representación más próxima a lo que un jurista intuitivamente llamaría contradicción entre normas estaría dado por esquemas del tipo $(\mathrm{A}>\mathrm{Op}) \mathrm{y}(\mathrm{B}>\mathrm{O}-\mathrm{p})$, normas que resultan incompatibles en el caso de la ocurrencia conjunta de sus antecedentes. Esto no es otra

${ }^{35}$ Puede consultarse el trabajo de Alchourrón citado en la nota anterior, así como «Philosophical Foundations of Deontic Logic and the Defeasible Conditionals», también citado.

${ }^{36}$ Alchourrón, Carlos y Bulygin, Eugenio, Introducción a la metodología de las ciencias jurídicas y sociales, ya citado, pág. 122.

${ }^{37}$ En el trabajo aludido en la cita anterior, pág. 102. 
cosa que decir, con otras palabras, algo que ya expresamos: un sistema de normas puede ser inconsistente con relación a ciertos hechos y no serlo frente a otros.

Lo dicho tiene importantes efectos en relación a las consecuencias que se deducen de la existencia de una contradicción en un sistema normativo. Pues es correcto afirmar que de dos enunciados contradictorios cualquier enunciado es consecuencia, por aplicación del principio de Duns Escoto:

$$
(\mathrm{A} \wedge-\mathrm{A})>\mathrm{B}
$$

Pero tratándose de dos enunciados condicionales, lo que se deduce de la contradicción entre sus consecuentes no es cualquier enunciado, sino la negación de su antecedente:

$$
[\mathrm{A}>(\mathrm{B} \wedge-\mathrm{B})] \vdash-\mathrm{A}^{38}
$$

Ello significa, en lo concerniente a los sistemas normativos, que si un caso está correlacionado con dos soluciones lógicamente incompa-

${ }^{38} \mathrm{Si}$ tomamos dos enunciados normativos condicionales cuyos antecedentes describen circunstancias fácticas y sus consecuentes son dos soluciones normativas que se contradicen [(A > Op) y $(\mathrm{B}>\mathrm{O}-\mathrm{p})]$, el esquema trascripto nos obligaría a aceptar la negación de la conjunción de los antecedentes:

$$
[(\mathrm{A} \wedge \mathrm{B})>(\mathrm{Op} \wedge \mathrm{O}-\mathrm{p})], \vdash-(\mathrm{A} \wedge \mathrm{B})
$$

Esto implicaría admitir que un sistema normativo con enunciados condicionales contradictorios posee consecuencias fácticas, cosa que es pacíficamente aceptada por Alchourrón y Bulygin en Introducción a la metodología de las ciencias jurídicas y sociales, ya citado (ver pág. 102). Pero tal conclusión es inadmisible pues significaría nada menos que caer en un vedado salto lógico de lo prescritivo a lo descriptivo, es decir, en la llamada falacia naturalista. Si me encuentro con mi auto frente a un semáforo rojo junto a una zona militar, ¿qué debo deducir de la contradicción entre las dos normas? ¿Acaso que no estoy frente a un semáforo rojo junto a una zona militar?

La respuesta a este nuevo problema, al igual que la correspondiente al tema del trabajo, es dificultosa pero en absoluto imposible. Requiere sí una profundización en el análisis lógico que trasciende el limitado cometido de estas páginas, por lo que diremos solamente que, a nivel sintáctico, la cuestión está estrechamente vinculada con la representación de las normas condicionales. Si utilizamos para su formalización conectivas debilitadas como los condicionales derrotables, la dificultad no surge por cuanto respeto de ellos no vale la ley de contraposición $[(\mathrm{A}>\mathrm{B}), \vdash(-\mathrm{B}>-$ A)]. Si en cambio empleamos el simple condicional material, será menester incluir entre las reglas de formación de nuestro sistema una flirtitación que bloquee la posibilidad de deducir consecuencias fácticas a partir de las soluciones normativas. Cualquiera de estas alternativas teóricas permitirá un adecuado acercamiento de la extensión del explicatum a la del explicandum. 
tibles, entonces ese caso está correlacionado con cualquier solución: todas las conductas son exigidas por el sistema frente al acaecimiento de las circunstancias de hecho que definen ese caso. Pero no es cierto que el sistema correlacione cualquier otro caso con cualquier solución. Anticipémonos a las críticas. Podría responderse a lo aquí afirmado que hemos restringido nuestra caracterización de la noción de contradicción normativa, identificándola exclusivamente con la contradicción entre las soluciones normativas de normas condicionales. Además, podrá argumentarse, considerar a la representación condicional como la más adecuada para los enunciados de un sistema jurídico no implica que sea la única viable, y negarse a aceptar tal reconstrucción permitiría eludir nuestra objeción. Finalmente, nada tiene de imposible que en los sistemas jurídicos puedan existir normas contradictorias categóricas en cuanto a las circunstancias, en cuyo caso seguiría siendo válida esta estrategia de impugnación a la aplicación de los desarrollos de la lógica al derecho.

Recordemos en primer lugar que una caracterización de la inconsistencia entre normas condicionales no es más restringida sino más amplia y abarcativa que la noción de inconsistencia absoluta con relación a las circunstancias, por cuanto la primera es una generalización de la segunda.

En segundo término, no se ha afirmado aquí en ningún momento que la representación condicional de los enunciados normativos sea la única viable, sino más bien lo contrario. Sí creemos, en cambio, haber dado elementos de juicio suficientes como para considerarla más adecuada, por lo menos frente a la reconstrucción en términos de normas categóricas con relación a las circunstancias. Su mayor virtualidad explicativa de los problemas que se presentan en la práctica con los sistemas jurídicos y la mayor intuitividad de sus aplicaciones es quizás susceptible de ser alcanzada o incluso superada por otras alternativas teóricas. Pero ello no quita que desde otras perspectivas de análisis también pueda brindarse una explicación satisfactoria de las consecuencias que intuitivamente los juristas asignan a las contradicciones entre normas.

Por último, ya fue reconocida expresamente la posibilidad de que un sistema jurídico contenga dos normas categóricas en cuanto a las circunstancias que sean incompatibles, en cuyo caso toda conducta sería exigible en cualquier circunstancia. Pero aún en el caso de que existiese un sistema tal, lo que parece poco probable, el mismo seguramente contendría otros enunciados normativos. Pongamos como ejemplo un modelo simplificado de sistema de tales características que contuviera cuatro normas, las dos primeras estableciendo la obligación de hacer $\mathrm{p}$ y de abstenerse de realizar $\mathrm{p}$ para toda circunstancia de hecho y las dos restantes estableciendo cada una la obliga- 
ción de realizar diferentes actos frente a distintas circunstancias particulares. Se podría en principio interpretar dichas normas de la siguiente manera:

$$
\begin{aligned}
& \text { N1: Op } \\
& \text { N2: O - p } \\
& \text { N3: } \text { > Oq } \\
& \text { N4: B > Or }
\end{aligned}
$$

En tal caso, resultaría que N3 y N4 son absolutamente superfluas, pues ellas se deducen de las dos primeras, tanto como también podrían deducirse:

$$
\begin{aligned}
& \mathrm{N} 5: \mathrm{A}>\mathrm{O}-\mathrm{q} \\
& \mathrm{N} 6: \mathrm{B}>\mathrm{O}-\mathrm{r}
\end{aligned}
$$

O cualquier otra. Pero ésta no es la única interpretación posible de las normas del sistema. Y seguramente no es la forma en que serían interpretadas por los desafortunados juristas que debieran trabajar con ellas. Una alternativa, seguramente más aceptable, sería entender que el legislador, al prever ciertas soluciones particulares para ciertos casos -N3 y N4-, ha querido establecer excepciones respecto de las obligaciones categóricas. En tal interpretación podría reconstruirse el sistema del siguiente modo:

$$
\begin{aligned}
& \text { N1: }-(\mathrm{A} v \mathrm{~B})>\mathrm{Op} \\
& \text { N2: }-(\mathrm{A} v \mathrm{~B}) \mathrm{O}-\mathrm{p} \\
& \text { N3: }>\text { Oq } \\
& \text { N4: B > Or }
\end{aligned}
$$

Aquí cobran sentido las normas N3 y N4, mientras que permanecen siendo exigibles todas las conductas en los casos en que no se da la circunstancia A ni la circunstancia B. El sistema así interpretado no deja de ser una pesadilla, pero se resguarda un marco de aplicación para las normas en las que el legislador ha previsto soluciones normativas particulares frente a ciertos hechos, con lo que N3 y N4 pueden perfectamente cumplir con su función de regular la conducta de sus destinatarios.

En resumen, la premisa del argumento de impugnación a la concepción deductivista que sostiene que en los sistemas jurídicos con enunciados contradictorios toda norma es derivable, se evidencia así como falsa, si se interpreta por «enunciados contradictorios» aquellos que establecen soluciones incompatibles con relación a ciertas circunstancias. Si, en cambio, entendemos por «enunciados contra- 
dictorios» una referencia a normas categóricas contradictorias, entonces podrá resultar verdadera, pero habrá que demostrar no sólo que los sistemas jurídicos existentes contienen enunciados contradictorios de esa clase, sino además que las restantes normas del sistema no pueden interpretarse como excepciones respecto de las normas categóricas contradictorias en cuestión, excepciones que dejarían sin efecto la obligatoriedad de todas las conductas para los casos de ocurrencia de sus antecedentes particulares. En todo caso, una hipótesis tal no es muy fácilmente conciliable con las habituales intuiciones de los juristas ni con las nuestras.

Igualmente ambiguo es afirmar que los sistemas jurídicos inconsistentes no cumplen con su función. La existencia de soluciones normativas contradictorias respecto de un cierto caso provoca que el sistema resulte inservible respecto de ese caso, sin que ello tenga incidencia alguna en las soluciones previstas para los restantes. Por tal razón, el sistema puede seguir cumpliendo su finalidad de regular la conducta de sus destinatarios respecto de cualquier otra circunstancia que no sea aquélla en la cual las soluciones se contradicen. $\mathrm{Y}$ esa es justamente la idea intuitiva que cualquier jurista tiene del efecto de una contradicción en un sistema jurídico.

La ambigüedad de estas premisas priva de toda eficacia al argumento en cuestión y deja sin sustento tanto a la tesis fuerte como a la tesis débil. Solamente una concepción deductivista ingenua de los sistemas jurídicos, que interpretara como categóricas a todas sus normas, sería impugnable por sus contraintuitivas implicancias. Pero en tal hipótesis, el problema no se encontraría en concebir al sistema como deductivo, sino en pretender reducir el intrincado tejido de enunciados jurídicos diversos a normas categóricas.

12. ¿Cuáles son entonces los límites en la aplicación de los desarrollos de la lógica al ámbito jurídico? Creemos que a estas alturas, los logros alcanzados han revertido los términos de la disputa: siendo que el estatus epistemológico de los estudios del derecho ha estado siempre en tela de juicio, y dado que la perspectiva lógica le ha posibilitado contar con un marco teórico poderoso y de incuestionable rigor, quien pretenda hoy descalificar al análisis lógico lleva sobre sus espaldas la carga de la prueba. Y al menos por lo que respecta a los argumentos que han sido tratados en el presente trabajo, no queda ni con mucho acreditado que sea necesario desplazar a la lógica formal o complementarla con otras misteriosas metodologías ${ }^{39}$.

${ }^{39}$ La recurrencia de nuestras citas a los trabajos de Alchourrón y Bulygin no es casual. Con ello hemos querido poner de manifiesto que, contrariamente a lo que 
Seguramente haya cosas en la vida -algunas al menos- para las cuales la lógica no sirva. Que el análisis lógico no agote ni con mucho las diversas perspectivas desde las cuales pueden fructíferamente estudiarse los fenómenos jurídicos es una verdad de Perogrullo, desde que ninguna disciplina científica conlleva semejante pretensión ${ }^{40}$. Pero no es necesario, para quien prefiera transitar por otras alternativas metodológicas, demostrar que la lógica no sirve. Que cada quien atienda a su juego.

expresa Atienza en su artículo referenciado en nota 1, en toda la obra de los profesores argentinos pueden hallarse extensas consideraciones explícitas o implícitas en réplica al argumento que hemos evaluado.

${ }^{40}$ Es real que la lógica aplicada al derecho representa un nivel de abstracción tal que, por cierto, no permite dar cuenta de ciertos aspectos de su dinámica. Un sistema jurídico puede ser concebido, por ejemplo, como un reflejo del tejido de relaciones de poder imperantes en una cierta comunidad social. Desde una postura como ésta, la lógica representará probablemente un criterio insuficiente para el estudio de los sistemas jurídicos. Resulta por lo menos paradójico, sino francamente incomprensible, que muchos críticos, pese a compartirla, no asuman directamente una posición como la antedicha para poner de relieve los límites del análisis lógico del derecho, y en cambio pretendan justificarlos valiéndose de argumentos lógicos. 